\title{
SOME REMARKS ON RELATIVELY COMPLETE FIELDS
}

\author{
IRVING KAPLANSKY AND O. F. G. SCHILLING
}

In this note we discuss some properties of fields which are relatively complete with respect to valuations of rank one. We show that relatively complete fields have in many respects simpler properties than complete fields. This note arose as a consequence of a discussion of an erroneous statement in a note of one of the authors. ${ }^{1}$

Let $K$ be a field on which a valuation $V$ of rank one is defined. Denote the ring of integers in $K$ with respect to $V$ by $O$ and let $P$ be the prime ideal of nonunits in $O$.

DEFINITION $^{2}$ 1. The field $K$ is termed relatively complete with respect to $V$ if every polynomial congruence

$$
f(x) \equiv g_{0}(x) h_{0}(x)(\bmod P)
$$

with $f(x)$ in $O[x]$ and $\left(g_{0}(x), h_{0}(x)\right) \equiv 1(\bmod P)$ implies

$$
f(x)=g(x) h(x)
$$

where $g(x) \equiv g_{0}(x)(\bmod P)$ and $h(x) \equiv h_{0}(x)(\bmod P)$.

We remark ${ }^{3}$ that there exist relatively complete fields which are not complete with respect to the congruence topology induced by $V$. To find examples for such fields it suffices to consider infinite algebraic extensions of a field which is complete with respect to a discrete valuation of rank one.

DEFINITION ${ }^{4}$ 2. Two polynomials $a(x)$ and $b(x)$ over a field $K$ are said to have the same decomposition type if $a(x)=\prod_{i=1}^{s} p_{i}(x)^{m_{i}}$, $b(x)=\prod_{i=1}^{r} q_{i}(x)^{n_{i}}$ when the $p_{i}(x)$ and $q_{i}(x)$ are irreducible polynomials and $s=r, m_{i}=n_{i}$ and $p_{i}(x)$ and $q_{i}(x)$ have the same degree for a suitable arrangement of the prime factors.

We are now able to generalize certain of the results of $F$. K.

Presented to the Society, December 31, 1941; received by the editors January 9 , 1942.

${ }^{1}$ O. F. G. Schilling, Remarks on a special class of algebras, American Journal of Mathematics, vol. 62 (1940), pp. 346-352.

${ }^{2}$ A. Ostrowski, Untersuchungen zur arithmetischen Theorie der Körper, Mathematische Zeitschrift, vol. 39 (1935), pp. 269-404.

${ }^{3}$ A. Ostrowski, Über einige Fragen der allgemeinen Körpertheorie, Journal für die reine und angewandte Mathematik, vol. 143 (1913), pp. 255-284.

${ }^{4}$ F. K. Schmidt, Mehrfach perfekte Körper, Mathematische Annalen, vol. 108 (1933), pp. 1-25. 
Schmidt. We first state without proof a simple consequence of Ostrowski's criterion for relative completeness. ${ }^{\mathbf{5}}$

LEMMA 1. Let $K$ be a field which is relatively complete with respect to a rank one valuation $V$ and let $\bar{K}$ be the completion of $K$. Then the decomposition type of any separable polynomial $f(x)$ with coefficients in $K$ is the same in $K$ as in $\bar{K}$.

LEMMA $^{6}$ 2. Let $\bar{K}, \bar{K}_{1}$ be the completions of $K$ with respect to the inequivalent rank one valuations $V, V_{1}$ of $K$, and let the fields $L, L_{1}$ be relatively complete with respect to $V$ and $V_{1}$ such that $K \leqq L \leqq \bar{K}, K \leqq L_{1} \leqq \bar{K}_{1}$. Then there exists for any two separable polynomials $g(x), g_{1}(x)$ of the same degree in $L, L_{1}$ a third polynomial $h(x)$ in $K$ which has the same decomposition types as $g(x), g_{1}(x)$ in $L, L_{1}$.

Proof. According to a result of F. K. Schmidt ${ }^{7}$ there exists a polynomial $h(x)$ in $K$ whose decomposition types in $\bar{K}, \bar{K}_{1}$ coincide with those of $g(x), g_{1}(x)$. Then, by Lemma 1, the decomposition types of $h(x)$ and $g(x)$ are the same in $L$. Similarly, the decomposition types of $h(x)$ and $g_{1}(x)$ are the same in $L_{1}$.

Next we prove the following theorem. ${ }^{8}$

THEOREM 1. If the field $K$ is relatively complete with respect to the rank one valuation $V$ and if, for the inequivalent rank one valuation $V_{1}$, the field $L_{1}$ is a relatively complete field containing $K$ and contained in the completion of $K$, then $L_{1}$ is separably algebraically closed.

Proof. Let $g_{1}(x)$ be an irreducible separable polynomial of degree $n$ in $L_{1}$ and let $g(x)$ be the product of any $n$ distinct linear factors in $K$. Then, according to Lemma 2, the polynomials $g(x)$ and $g_{1}(x)$ have the same type of decomposition in $K$ and $L_{1}$. Hence $g_{1}(x)$ must be a product of linear polynomials; in other words, the field $L_{1}$ is separably algebraically closed.

THEOREM 2. A field $K$ is relatively complete with respect to two inequivalent rank one valuations if and only if it is separably algebraically closed.

Proof. The necessity of the statement follows at once from Theorem 1. On the other hand Ostrowski's criterion implies that a separa-

\footnotetext{
${ }^{5}$ Ostrowski's criterion states that a field is relatively complete if and only if it is separably algebraically closed in its complete closure. Loc. cit., p. 316.

${ }^{6}$ F. K. Schmidt, loc. cit., lemma on p. 16.

7 Loc. cit., p. 16.

8 Theorem 1 is the analogue of Schmidt's Theorem 1, p. 18, loc. cit.
} 
bly algebraically closed field is relatively complete with respect to every valuation of rank one.

THEOREM 3. If a field $K$ is complete with respect to a rank one valuation and relatively complete with respect to a rank one valuation which is inequivalent to the first valuation, then $K$ is a multiply complete field.

Proof. By Theorem 2 the field $K$ is separably algebraically closed. Since $K$ is complete with respect to the second valuation, it follows that $K$ is algebraically closed. ${ }^{9}$ Moreover the cardinal number $\mathfrak{c}$ of $K$ satisfies the equation $\mathfrak{c}^{\boldsymbol{N}_{0}}=\mathfrak{c}$ for $K$ is a complete field. Hence $K$ is a multiply complete field.

We should like to point out that the above theorems do not require conditions on the cardinal number of the field $K$ as in the theory of F. K. Schmidt for complete fields.

In Theorem 2 it was proved that the separable algebraic closure of a field $K$ is always relatively complete with respect to every valuation extending any non-trivial rank one valuation of $K$. We then may ask whether already a finite algebraic extension $L$ of $K$ is relatively complete with respect to some rank one valuation though $K$ itself is not relatively complete with respect to any rank one valuation.

We shall give a partial answer to this problem in this theorem.

THEOREM 4. Suppose that $K$ is a field which has for every integer $n$ exactly one separable extension of degree $n$ and suppose that $K$ is not relatively complete with respect to any non-trivial rank one valuation. Then there exists no finite algebraic extension $L$ of $K$ which is relatively complete with respect to a rank one valuation.

Proof. Suppose that, contrary to the theorem, there exists a finite extension $L$ of degree $m$ over $K$ which is relatively complete with respect to some rank one valuation $V_{L}$. Since $L$ is relatively complete with respect to $V_{L}$ there is, by Ostrowski's criterion, a one-to-one correspondence between the algebraic extensions of $L$ and its completion $\bar{L}$ with respect to $V_{L}$. In other words, if $Z_{n}$ is an arbitrary extension of degree $n$ over $L$ then $\left[Z_{n} \bar{L}: \bar{L}\right]=n$. Consider next the valuation $V$ on $K$ which is induced by $V_{L}$. Let $\bar{K}$ be the completion of $K$ with respect to $V$. We assert that the Galois group of the algebraic closure $\bar{K}^{\prime}$ of $\bar{K}$ (relative to $\bar{K}$ ) is cyclic. For a proof it suffices to show that the decomposition groups for the prolongations $V_{i}$ of $V$ to any extension $M>K$ are cyclic. ${ }^{10}$ Since $M / K$ is cyclic all decom-

${ }^{9}$ F. K. Schmidt, loc. cit. Lemma 2, p. 18.

${ }^{10} \mathrm{M}$. Deuring, Verzweigungstheorie bewerteter Körper, Mathematische Annalen, vol. 105 (1931), pp. 277-307. 
position fields coincide and are cyclic. The field $\bar{L}$ is then equivalent to a subfield of $\bar{K}^{\prime}$; without loss of generality we may suppose $\bar{K}^{\prime}>\bar{L} \geqq \bar{K}$. The degree $[\bar{L}: \bar{K}]=\bar{m}$ is a divisor of $m$. Consequently $\left[Z_{n} \bar{L}: \bar{K}\right]=\left[Z_{n} \bar{L}: \bar{L}\right][\bar{L}: \bar{K}]=n \bar{m}$. By the Galois theory there is then for every integer $n$ an extension $Z_{n}^{*}$ of degree $n$ over $\bar{K}$. The defining equation $f^{*}(x)=0$ of $Z_{n}^{*} / \bar{K}$ now may be approximated by an irreducible equation $f(x)=0$ of degree $n$ with coefficients in $K$ so that $Z_{n}^{*}$ is generated by the roots of $f(x)=0$. The root field of $f(x)=0$ over $K$ is the cyclic extension $Z_{n}^{\prime}$ of degree $n$ over $K$. Hence $Z_{n}^{*}=Z_{n}^{\prime} \bar{K}$ for all $n$, contrary to the assumption that $K$ is not relatively complete with respect to any rank one valuation.

HARVARD UNIVERSITY AND

University OF CHICAGO

\section{A DIFFERENTIAL GEOMETRY PROBLEM USING TENSOR ANALYSIS}

ATHERTON H. SPRAGUE

1. Introduction. The problem at hand was worked out in attempting to apply tensors to a much more general problem in classical differential geometry. The results obtained in a general coordinate system reduce readily to classical results of Eisenhart. An interesting interpretation of Christoffel symbols appears.

2. $R$ net. A rectilinear congruence in 3 -space is called a $W$-congruence if the asymptotic lines on the two focal surfaces correspond. If the tangents to both families of curves of a conjugate net on a surface form $W$-congruences the net is called an $R$ net. ${ }^{1}$ We derive the analytic conditions that must obtain in order that a given conjugate net on a surface shall be an $R$ net.

3. Equations for an $R$ net. Let $S_{1}$ be one focal surface of a $W$-congruence, the vector equation of the surface being

$$
z_{1}^{\alpha}=z_{1}^{\alpha}\left(x^{i}\right), \quad \alpha=1,2,3 ; i=1,2 .
$$

Received by the editors November 11, 1941.

${ }^{1}$ Tzitzeica, Comptes Rendus de l'Académie des Sciences, Paris, vol. 152 (1911), p. 1077. 